\title{
Análise da cicatrização do cólon com uso do extrato aquoso da Orbignya phalerata (Babaçu) em $\operatorname{ratos}^{1}$
}

\author{
Healing of colonic anastomosis with the use of extract aqueous of Orbignya phalerata \\ (Babassu) in rats
}

\begin{abstract}
Raimundo Nonato Baldez ${ }^{2}$, Osvaldo Malafaia ${ }^{3}$, Nicolau Gregori Czeczko ${ }^{3}$, Nelson Lúcio Parada Martins ${ }^{2}$, Lydia Masako Ferreira ${ }^{3}$, Carmen Australia Paredes Marcondes Ribas ${ }^{3}$, Guataçara Salles Júnior ${ }^{4}$, Rafael Pasini Del Claro ${ }^{4}$, Luciana de Oliveira Marques dos Santos ${ }^{4}$, Lincoln Graça Neto ${ }^{5}$, Luiz Roberto Reis de Araújo ${ }^{4}$
\end{abstract}

1. Trabalho realizado no laboratório de Pesquisas do Centro de Ciências Biológicas e da Saúde da Universidade Federal do Maranhão

2. Professor do Departamento de Cirurgia da Universidade Federal do Maranhão

3. Professor Doutor em Cirurgia

4. Aluno de Pós-Graduação - Mestrado

5. Aluno de Pós-Graduação - Doutorado

\section{RESUMO}

Introdução: A cicatrização é processo complexo, com diferentes fases. Embora exista grande variedade de plantas medicinais poucas têm sido investigadas com intenção de melhorar a cicatrização de órgãos e tecidos, dentre elas, a Orbignya phalerata (Babaçu). Objetivo: Analisar comparativamente as alterações tensiométricas e histológicas na cicatrização de anastomoses colônicas proporcionadas pelo uso do extrato aquoso do mesocarpo do Babaçu (Orbignya phalerata). Metodos: Utilizaramse 40 ratos Wistar, machos, separados em dois grupos de vinte, denominados grupos controle e experimento, Os ratos de cada grupo foram divididos em dois sub-grupos de 10 animais cada e avaliados no terceiro e sétimo dia de pós-operatório. Realizaram-se colotomia e colorrafia em plano único. Os animais do grupo controle receberam no ato operatório dose única de solução salina isotônica em dose e via de administração semelhantes ao grupo experimento e os do grupo experimento receberam extrato aquoso de Babaçu em dose de $50 \mathrm{mg} / \mathrm{kg} /$ peso, concentração de $25 \mathrm{mg} / \mathrm{ml}$ por via intraperitoneal. Foram analisados os seguintes parâmetros: a) avaliação macroscópica da parede e cavidade abdominal; b) avaliação tensiométrica da anastomose (pressão e ruptura); c) características histológicas da anastomose. Resultados: O exame macroscópico evidenciou a presença de aderências em todos os animais, sendo estatisticamente maior no grupo experimento de três dias quando comparados com o grupo controle. Nos animais do grupo de sete dias não houve diferença. Não houve ocorrência de deiscência de anastomose, fístulas, abscessos e/ou hemorragia. Com relação à tensiometria, constatou-se que a média de ruptura dos grupos controle e experimento tanto para os animais do grupo de três dias $(25,4 \mathrm{mmHg}-14,8 \mathrm{mmHg})$ quanto para os de sete dias $(183 \mathrm{mmHg}-175 \mathrm{mmHg})$, foram iguais, não ocorrendo diferença estatisticamente significante. A análise histológica dos animais do grupo de três dias mostrou diferença estatisticamente significante no grupo experimento em relação ao controle na variável mononuclear, enquanto que, no sétimo dia, houve diferença estatisticamente significante favorável ao grupo experimento em todas as variáveis quando comparadas com o de controle. Conclusão: Ocorreu efeito favorável no uso do extrato aquoso do mesocarpo do Babaçu no processo de cicatrização da anastomose colônica, não havendo diferença significante na avaliação tensiométrica entre os grupos controle e experimento.

Descritores: Cicatrização de Feridas. Gorduras Vegetais. Anatomoses Cirúrgicas. Cólon. Ratos Wistar.

\begin{abstract}
Introduction: Healing is a highly complex process with different phases. Because some medicinal plants have antiinflamatory properties, they could influence the wound healing process. There are many species of medicinal plants used for research, among them the Orbignya phalerata (Babassu). Purpose: To analyze, comparatively, the tensiometric and histologic alterations in the healing process of colon anastomosis with the use of aqueous extract of the Babaçu mesocarp. Methods: Forty Wistar male rats were used, divided in two groups of 20 animals named control and experimental group. Each group was further divided in two sub-groups of 10 animals and evaluated on the third and seventh post-operative days. Colotomy and one suture line coloraphy was performed in all animals. The control group received an intraperitoneal single dose of isotonic saline solution at the time of the surgery. The animals from the experimental group received babassu aqueous extract dose with $50 \mathrm{mg} / \mathrm{kg} /$ weight, concentration of $25 \mathrm{mg} / \mathrm{ml}$ dose, with the same administration method. The following parameters were evaluated: a) macroscopic appearance of abdominal wall and cavity; b) tensiometric evaluation of anastomosis (bursting pressure); c) histologic characteristics of anastomosis. Results: The macroscopic examination showed the presence of adherences in all animals with statistical significance in the ones from the experimental group at third day when compared to the control group. No difference was observed in the seventh-day group. No fistulae, abscesses and/or hemorrhage were observed. In regard to the tensiometric variation, the bursting pressure for the control
\end{abstract}


and experimental groups, both for the third day $(25.4 \mathrm{mmHg}-14.8 \mathrm{mmHg})$ and at seventh $(183 \mathrm{mmHg}-175 \mathrm{mmHg})$, were similar with no significant difference. Histological analysis for the third day showed significant difference in the experimental group in relation to the controls, in regard the presence ofto mononuclear ones whereas at seventh day there was significant statistical difference in all variables from the experimental group compared to the control one. Conclusion: A favorable effect with administration of the aqueous extract of the Babaçu mesocarp was seen in histological parameters of the healing process of colon anastomosis however there was no difference in the tensiometric evaluation between the control and experimental group.

Key words: Wound Healing. Vegetable Fats. Anastomosis Surgical. Colon. Wistar Rats.

\section{Introdução}

A cicatrização é evento biológico complexo envolvendo inflamação, quimiotaxia, proliferação celular, diferenciação e remodelação ${ }^{1}$. A despeito dos diversos métodos de avaliação, do ponto de vista morfológico, após uma lesão, identificam-se três fases consecutivas do processo de reparação: inflamatória ou inicial; fibroplasia ou proliferativa e maturação ou remodelação. Cumpre, entretanto, salientar que tais fases não constituem processos isolados, e sim sinergismo visível, que, com freqüência, sobrepõe uma fase na outra de tal sorte que, em uma mesma fase, pode-se encontrar os elementos que compõem a subseqüente ${ }^{2}$.

Corsi et al. $^{3}$ consideram de fundamental importância o conhecimento da intimidade de cada uma dessas fases, pois isto possibilitaria a aplicação de condutas terapêuticas adjuvantes à reação biológica para reparação tecidual, tão correta quanto possível.

O processo de cicatrização pode sofrer bloqueio ou retardo em função de diversos fatores locais e sistêmicos ${ }^{12}$. Greca et al. ${ }^{3}$ estudando a interferência da inflamação na cicatrização concluem que a reação inflamatória aumenta o número de deiscências anastomóticas no cólon, provavelmente pelo atraso de maturação e ordenação do colágeno. Ainda os autores relatam maior concentração de colágeno maduro e total nas anastomoses colônicas pela ação de ácidos graxos de cadeia curta.

Vários estudos em animais de experimentação têm sido desenvolvidos para avaliação de fatores que possam interferir positiva ou negativamente no fenômeno da cicatrização, inclusive com a utilização de plantas medicinais que constituem a matéria prima para elaboração e preparação industrial de derivados químicos puros.

É importante enfatizar que algumas das plantas utilizadas pela população apresentam propriedades terapêuticas, mas também podem apresentar grande toxicidade, o que torna o seu uso indiscriminado fator de risco. Assim, o conhecimento específico sobre a atividade biológica, a identificação dos constituintes e compostos bioativos, bem como a determinação da concentração adequada, são etapas indispensáveis à sua correta utilização ${ }^{4}$.

O Babaçu é palmeira do reino vegetal, classe Monocotyledoneae, família Palmae, do gênero Orbignya, espécie Orbignya phalerata, nativa do meio norte brasileiro com sua maior incidência nos estados do Maranhão, Piauí, Tocantins, Goiás e Mato Grosso e tem sido popularmente utilizada como alimento ou remédio ${ }^{5}$.

Seu mesocarpo moído é o mais utilizado tanto na alimentação como no receituário caseiro ${ }^{6}$.

Em geral, a farinha dele obtida é utilizada na medicina dentre outras indicações como antiinflamatório ${ }^{7}$.

$\mathrm{Na}$ literatura médica, encontra-se número reduzido de trabalhos experimentais com relação ao Babaçu. Dentre eles, MAIA ${ }^{8}$, realizando estudo experimental para testar sua possível atividade antiinflamatória, concluiu que na forma de extrato clorofórmico, ela promove moderada ação analgésica sem efeitos narcóticos. No entanto, Chagas ${ }^{9}$ realizou outro experimento concluindo não existir, nas doses por ele utilizadas, atividade analgésica ou antiinflamatória.

Procurando ajudar a esclarecer esta controversa na área da fitoterapia brasileira, o presente estudo tem por objetivo analisar comparativamente os achados tensiométricos e as alterações histológicas das anastomoses colônicas quando submetidas à influência do extrato aquoso do Babaçu.

\section{Métodos}

Este estudo foi realizado no Laboratório de Pesquisa do Departamento de Fisiologia e Farmacologia do Centro de Ciências Biológicas e da Saúde da Universidade federal do Maranhão - UFMA respeitando-se os princípios éticos de experimentação animal recomendados pelo Colégio Brasileiro de Experimento Animal e aprovado pelo Comitê de Ética da UFMA.

Foram utilizados 40 ratos (Rattus novergicus albinus) machos, adultos, da linhagem Wistar, com peso corpóreo variando entre 107 e 183 gramas, com idade de 60 dias, procedentes do Biotério do Centro Multidisciplinar para Investigação Biológica da Universidade Estadual de Campinas - CEMIB - UNICAMP . Eles foram alojados em gaiolas coletivas contendo cinco animais, sob condições de temperatura e umidade ambientais, em ciclo circadeano dia e noite normal, recebendo ração padrão Purina-Labina ${ }^{\circledR}$ e água ad libitum livre e mantendo-se período de sete dias para aclimatação.

A identificação deles foi feita com ácido pícrico em quatro pontos : cabeça; cabeça e dorso; dorso; cauda. As gaiolas continham etiqueta com o nome do pesquisador, tipo da planta, datas da operação e da eutanásia. Realizou-se pesagem diária dos animais, inclusive do dia da eutanásia, com registro em ficha protocolo.

Foram padronizados dois grupos aleatoriamente constituídos e compostos por 20 animais cada, denominados de grupos controle e experimento. Cada um foi dividido em dois subgrupos de 10 animais, para serem avaliados no $3^{\circ} \mathrm{e}$ $7^{\circ}$ dias de pós-operatório.

Os animais do grupo experimento (GE) foram submetidos ao tratamento com solução de extrato aquoso do Babaçu na dose de $50 \mathrm{mg} / \mathrm{Kg}$ (concentração de $25 \mathrm{mg} / \mathrm{mL}$ ), por via intraperitoneal diretamente na cavidade. Nos animais do 
grupo controle (GC) utilizou-se solução salina a $0,9 \%$ em dose, com volume e via de administração idênticos ao grupo experimento.

\section{Preparo do extrato aquoso do mesocarpo}

A coleta do mesocarpo foi feita na área do Campus Universitário do Bacanga, São Luís do Maranhão e catalogada sob o número 1136, no Herbário Ático Seabra da UFMA.

A constituição química do produto é de amido (68,3\%), proteínas $(1,54 \%)$, lipídios $(0,27 \%)$, glicídios solúveis $(1,25 \%)$, fibras $(2,51 \%)$, umidade $(14,90 \%)$ e outras substâncias orgânicas como aminoácidos, hemicelulose e pentosanas $(11,23 \%)$.

O mesocarpo foi obtido a partir do coco maduro quando caído naturalmente do cacho. Para a retirada do fruto, utilizou-se artefato de madeira batendo-se na parte superior até a ruptura da casca. A seguir foi posto a secar naturalmente durante três dias e depois colocado em estufa de secagem complementar na temperatura de $45-50^{\circ} \mathrm{C}$ durante $24 \mathrm{~h}$. O mesocarpo foi então submetido à moagem elétrica, de onde se obteve pó em forma de farinha.

Para a preparação do extrato aquoso, o pó foi pesado e diluido em solução salina para concentração de $25 \mathrm{mg} / \mathrm{ml}$ (10g/400ml de solução salina).

\section{O experimento}

Após jejum de 12 horas, mantendo-se ingestão de água ad libitum, a indução anestésica foi realizada colocando-se o animal em campânula de plástico transparente contendo em seu interior chumaço de algodão embebido em éter sulfúrico comercial, permanecendo por tempo médio de cinco minutos, até a completa abolição do reflexo corneano. O animal anestesiado era retirado da campânula e posicionado em decúbito dorsal horizontal mantendo-se assim por inalação com éter sulfúrico via nasal, sendo o período anestésico e sua recuperação anotadas em ficha protocolo.

A técnica operatória foi a mesma para ambos os grupos realizando-se como preparo do campo operatório os princípios habituais de procedimento cirúrgico abdominal. A incisão foi mediana de $4 \mathrm{~cm}$ de extensão. Procedeu-se a seguir a secção transversal (colotomia) simples do cólon $2 \mathrm{~cm}$ acima da reflexão peritoneal e subsequënte anastomose término-terminal com seis pontos separados em plano único. Imediatamente após a realização da anastomose, os animais foram divididos de maneira aleatória, onde o primeiro foi escolhido por sorteio e os seguintes alternados em grupo controle e experimento.

O grupo experimento recebeu tratamento com solução de extrato aquoso do Babaçu na dose de $50 \mathrm{mg} / \mathrm{kg}$ (concentração de $25 \mathrm{mg} / \mathrm{ml}$ ), diretamente na cavidade peritoneal; o grupo controle, solução salina a $0,9 \%$ em quantidade e via de administração idênticas.

Realizou-se a síntese da parede abdominal em dois planos.

Após o ato operatório, os animais foram colocados em suas respectivas gaiolas, recebendo ração padrão e água ad libitum. Procedeu-se avaliação clínica (sítio cirúrgico, atividade motora, disposição para alimentação) e registro diário do peso corpóreo, inclusive no dia da eutanásia em protocolo próprio.

A eutanásia foi realizada por inalação de dose letal de éter sulfúrico. Dez animais de cada grupo foram sacrificados no $3^{\circ}$ e $7^{\circ}$ dias de pós-operatório. Após o óbito, a relaparotomia era realizada mediante duas incisões transversais de $3 \mathrm{~cm}$ (abaixo do apêndice xifoide e acima do púbis) e outra incisão vertical paralela à cicatriz cirúrgica prévia configurando um retalho cutâneo tipo folha de livro.

Além dos dados, obtinham-se fotos para registro das complicações relacionadas com o sítio cirúrgico, cavidade peritoneal e presença de aderências, quantificadas pelo grau de adesão em 0 - sem adesão; I - adesão única entre dois órgãos ou entre um órgão e a parede abdominal; II - duas adesões entre órgãos ou um órgão e a parede abdominal; III - mais de duas aderências entre órgãos ou uma massa de adesão generalizada no intestino sem aderir à parede abdominal; IV - aderências generalizadas entre órgãos e paredes maciças entre todos os órgãos.

A peça cirúrgica foi seccionada $2 \mathrm{~cm}$ acima e abaixo da linha da anastomose, compreendendo ceco, cólon ascendente e segmento de íleo terminal de $4 \mathrm{~cm}$ de comprimento. As estruturas aderidas ao cólon foram retiradas em monobloco.

Para avaliação tensiométrica, foi realizada a limpeza mecânica da peça cirúrgica com solução salina a 0,9\%, mantendo-se todas as aderências com o cólon. Após oclusão do íleo terminal mediante ligadura simples, introduziu-se sonda siliconizada $n^{\circ} 6$ na porção proximal do cólon ascedente (1cm) fixando-a nesse segmento com ligadura simples. A sonda era conectada à bomba de infusão com insuflação de ar de forma constante e contínua. A pressão do ar insuflado era registrado em milímetros de mercúrio, através de manômetro eletrônico.

Após esses procedimentos, a peça era submersa em recipiente de vidro transparente contendo água corrente e a resistência tênsil da anastomose era testada através da insuflação gradativa de ar $(10 \mathrm{mmHg} /$ segundo - velocidade $)$, até a ocorrência de borbulhamento na água evidenciando a ruptura da peça, registrando-se, nesse momento, o local e o nível de pressão determinantes da ruptura do cólon (Figura 1).

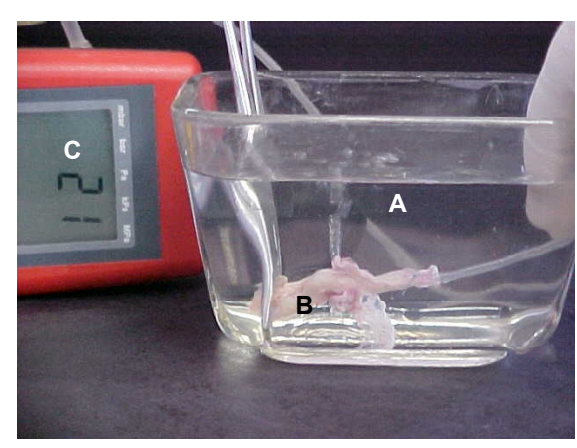

FIGURA 1 - Momento de ruptura da anastomose colônica. Nota: A - Recipiente de vidro transparente contendo água corrente; B - momento de ruptura da anastomose colônica evidenciada pelo borbulhamento na água; $\mathrm{C}$ - pressão de ruptura em $\mathrm{mmHg}$ 
As peças cirúrgicas foram encaminhadas para estudo histológico, realizado pelo mesmo patologista, sem o conhecimento prévio da identificação do grupo a que elas pertenciam. As lâminas foram coradas pelo método de Hematoxilina - eosina para avaliação global dos cortes de tecido e pelo Tricômio de Masson para demonstrar a presença de fibras colágenas existentes nos locais de fibrose do interstício

Para uniformização da análise histológica, observaramse os seguintes critérios: congestão vascular, edema, tipo de infiltrado inflamatório, proliferação fibroblástica, colagenização e angiogênese. Cada critério foi graduado de 0 a 3 da maneira seguinte: ausente 0 ; discreto 1; moderado 2 e acentuado 3 , utilizando-se protocolo de avaliação microscópica.

A análise histológica considerou o acometimento tissular pelo processo inflamatório quanto ao infiltrado inflamatório (intensidade, modo de distribuição no interstício e tipo de células presentes) e ao grau da fibrose intersticial.

Para avaliação numérica, a reação inflamatória foi considerada aguda entre -9 a -3 ; subaguda, entre $-2,9$ a $+2,9$; e crônica, entre $+3 \mathrm{a}+9$. Os parâmetros negativos correspondiam à congestão, edema e presença de polimorfonucleares e mensurados como ausente (0), discreta quantidade (-1), moderada quantidade (-2) e acentuada quantidade (-3). Os parâmetros positivos correspondiam à presença de células mononucleares, proliferação fibroblástica, angiogênese e colagenização e foram classificados como ausente $(0)$, discreta quantidade $(+1)$, moderada quantidade $(+2)$ e acentuada quantidade $(+3)$.

Os dados foram analisados estatisticamente utilizandose o programa Statistics for Windows 5.1 (1996). A pressão de insuflação de ar foi analisada pelo teste t de Student. As variáveis da avaliação microscópica e macroscópica foram analisadas pelo teste não-paramétrico de Mann-Whitney. O nível de significância (p) utilizados para se rejeitar a hipótese da nulidade foi de $5 \%(\mathrm{p}<0,05)$.

\section{Resultados}

\section{Avaliação macroscópica}

Todos os animais recuperaram-se bem da anestesia e a cicatrização da parede abdominal foi considerada boa em todos, com exceção de quatro (dois de cada grupo) que apresentaram infecção discreta no sítio cirúrgico e uma deiscência cutânea parcial no grupo experimento do $3^{\circ}$ dia.

Em relação à intensidade das aderências abdominais, no $3^{\circ}$ dia elas estiveram presentes no grupo controle em cinco animais com o tipo I; com o II em quatro; com o III em um animal. No grupo experimento elas estavam assim distribuídas: tipo II em quatro animais e tipo III, em seis.

Já no $7^{\circ}$ dia, nos animais do grupo controle constatou-se o tipo I em cinco animais; o II em três e o III em dois. No grupo experimento ocorreu o tipo I em um animal; o II em três; o III em cinco.

Com relação à análise macroscópica das variáveis deiscência e aderência nos animais dos grupos controle e experimento do $3^{\circ}$ dia, houve diferença estatisticamente significante entre elas (Tabela 1), enquanto que nos grupos controle e experimento do $7^{\circ}$ dia a variável aderência não foi estatisticamente significante em relação à deiscência (Tabela 2).

TABELA 1 - Resultado da análise macroscópica do $3^{\circ}$ dia

\begin{tabular}{lcccc}
\hline & $\begin{array}{c}\text { Grupo } \\
\text { Controle }\end{array}$ & $\begin{array}{l}\text { Grupo } \\
\text { Experimento }\end{array}$ & Z & p \\
\hline Deiscência & 110 & 100 & 0,378 & 0,705 \\
Aderência & 70 & 140 & $-2,646$ & $0,008^{*}$ \\
\hline $\begin{array}{l}\text { Z-teste estatístico } \\
\text { p }^{*} \text { - significância estatística }\end{array}$ & & & \\
\end{tabular}

TABELA 2 - Resultado da análise macroscópica do $7^{\circ}$ dia

\begin{tabular}{lcccc}
\hline & $\begin{array}{l}\text { Grupo } \\
\text { Controle }\end{array}$ & $\begin{array}{l}\text { Grupo } \\
\text { Experimento }\end{array}$ & $\mathbf{Z}$ & $\mathbf{p}$ \\
\hline Deiscência & 105 & 105 & 0 & 1 \\
Aderência & 88 & 122 & 1,285 & 0,199 \\
\hline
\end{tabular}

$\mathrm{Z}$ - teste estatístico

$\mathrm{p}^{*}$ - significância estatística

\section{Avaliação tensiométrica}

O teste de resistência à insuflação de ar atmosférico foi realizado em todos os 40 animais estudados. Observou-se que no $3^{\circ}$ dia pós-operatório o vazamento de ar ocorreu em todos os animais, na linha anastomótica; já no $7^{\circ}$ dia, $95 \%$ das vezes ocorreu distante dela.

Os valores médios da pressão de ruptura no grupo do $3^{\circ}$ dia foram para o grupo controle de $25,4 \mathrm{mmHg}$ e experimento de $14,8 \mathrm{mmHg}$. No de $7^{\circ}$ dia foram: grupo controle $183 \mathrm{mmHg}$ e experimento 175,6 mmHg (Figura 2).

O teste $t$ de Student usado para comparação das médias no teste de pressão ruptura de ar dos grupos do $3^{\circ} \mathrm{e}$ $7^{\circ}$ dias (controle e experimento) não revelou significância estatística, embora os níveis de pressão fossem significativamente maiores no $7^{\circ}$ dia (Tabela 3 ) em ambos os grupos em relação ao $3^{\circ}$. dia.

$\mathrm{Na}$ análise da homogeneidade das varianças, não foi observada diferença significativa entre a variança do grupo controle e experimento, tanto no $3^{\circ}(\mathrm{F}=2,082, \mathrm{p} .=0,290)$ como no $7^{\circ}$ dia $(\mathrm{F}=2,821, \mathrm{p}=0,138)$.

\section{Avaliação microscópica}

$\mathrm{Na}$ análise microscópica do $3^{\circ}$ dia (Figuras 3 e 4), houve predomínio absoluto da fase inflamatória aguda, tanto no grupo controle como no experimento, com exceção de um animal deste último grupo, que apresentou inflamação subaguda. Todos os animais do grupo controle e experimento apresentaram edema, congestão e polimorfonucleares, variando da forma leve, moderada e intensa. A proliferação 


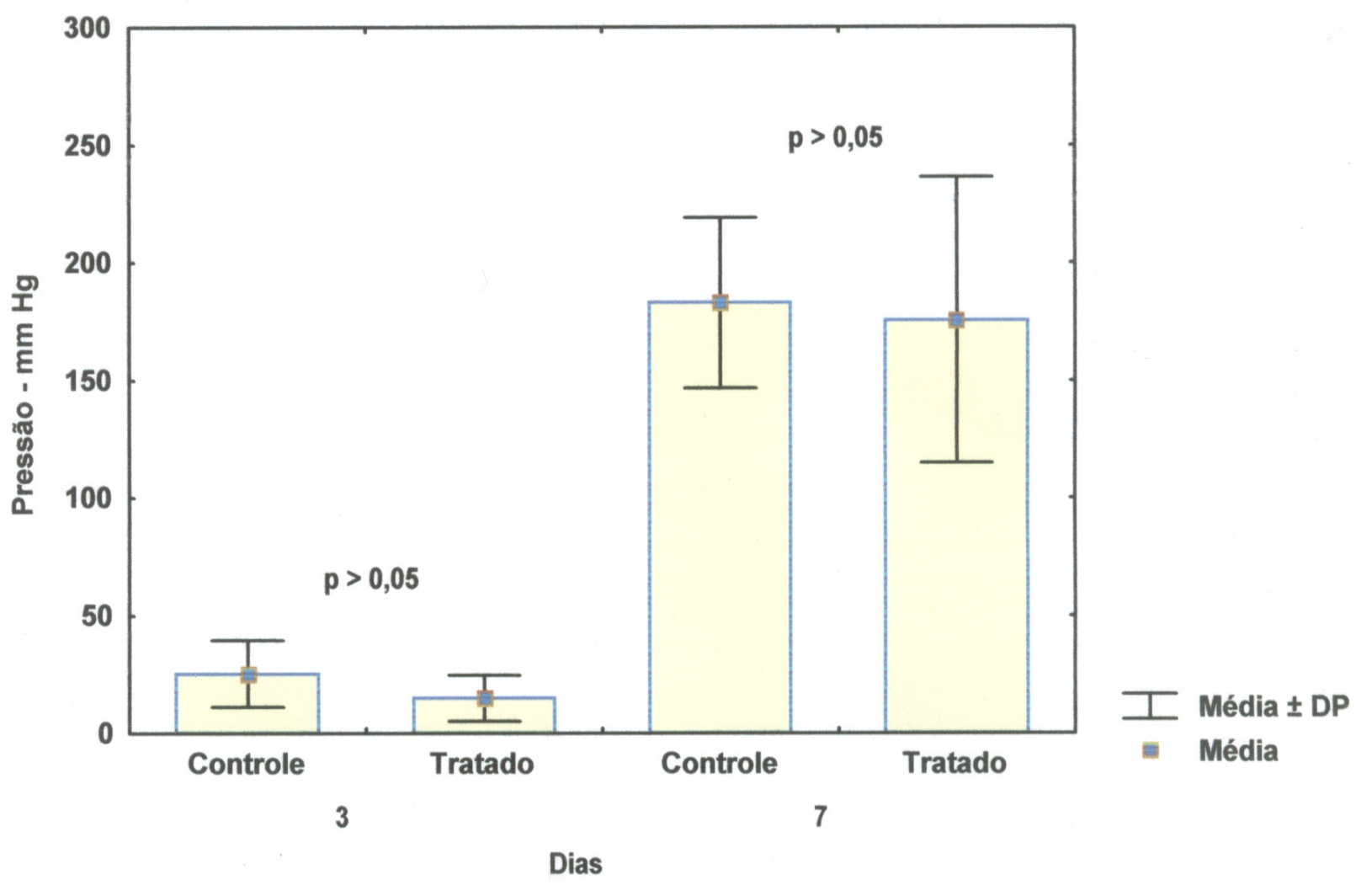

FIGURA 2 - Médias e desvio padrão dos grupos controle e experimento no teste de insuflação de ar do $3^{\circ}$ e $7^{\circ}$ dias

TABELA 3 - Comparação da média de pressão de ruptura das anastomoses colônicas após insuflação de ar atmosférico nos animais do grupo controle e experimento do $3^{\circ}$ e $7^{\circ}$ dias

\begin{tabular}{lcccc}
\hline & $\begin{array}{l}\text { Média Grupo } \\
\text { Controle }\end{array}$ & $\begin{array}{l}\text { Média } \\
\text { Grupo } \\
\text { Experimento }\end{array}$ & T & p \\
\hline 3 Dias & $25,4 \mathrm{mmHg}$ & $14,8 \mathrm{mmHg}$ & 1,949 & 0,067 \\
7 Dias & $183,0 \mathrm{mmHg}$ & $175,6 \mathrm{mmHg}$ & 0,331 & 0,741 \\
\hline
\end{tabular}

Nota: Teste $\mathrm{t}$ de Student, $\mathrm{t}=$ teste, $\mathrm{p}^{*}=0,05$

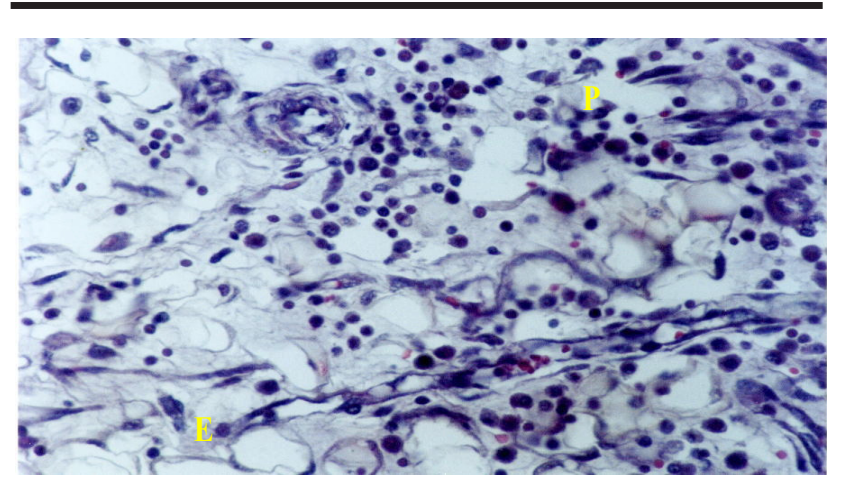

FIGURA 3 - Fotomicrografia de cólon do grupo controle no $3^{\circ}$ dia (Hematoxilina-Eosina, 400x). Nota: Observa-se inflamação aguda com edema (E) e polimorfonucleares $(\mathrm{P})$.

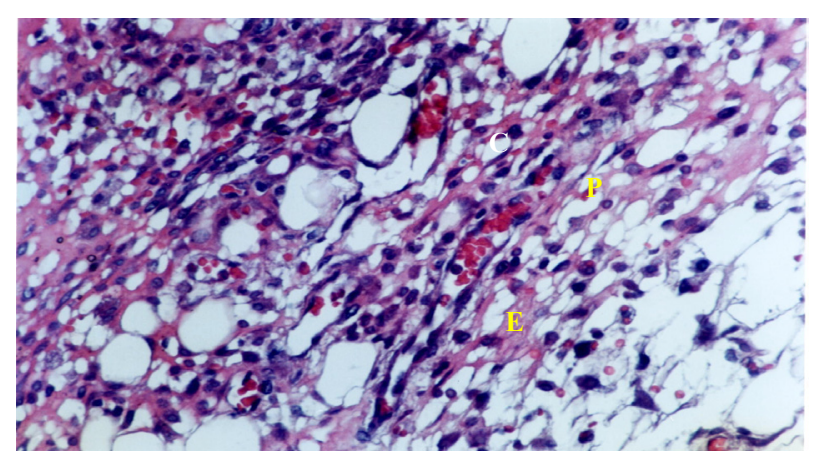

FIGURA4-Fotomicrografia de cólon do grupo experimento no $3^{\circ}$ dia (Hematoxilina-Eosina, 400x). Nota: Observa-se inflamação aguda com congestão (C), edema (E) e polimorfonucleares (P)

fibroblástica esteve presente somente em cinco animais do grupo experimento. A angiogênese foi demonstrada também em cinco animais do grupo experimento de forma leve. A colagenização foi ausente em todos os animais de todos os grupos, havendo diferença estatisticamente significante $(p=0,023)$ entre o grupo experimento em relação ao grupo controle na variável mononucleares (Tabela 4).

$\mathrm{Na}$ análise microscópica do $7^{\circ}$ dia (Figuras 5 e 6), houve predominância absoluta de inflamação crônica em todos os animais do grupo experimento em relação ao grupo controle com significância em todas as variáveis utilizadas como parâmetro histológico de cicatrização (Tabela 5). 
TABELA 4 - Variáveis da análise histológica do $3^{\circ}$ dia

\begin{tabular}{|c|c|c|c|c|c|c|c|c|c|c|c|c|}
\hline $\begin{array}{l}\text { Variável } \\
\text { histológica }\end{array}$ & Grupo & $\mathrm{R} 1$ & R2 & $\mathrm{R} 3$ & R4 & R5 & R6 & R7 & $\mathrm{R} 8$ & R9 & R10 & $\mathrm{P}$ \\
\hline \multirow[t]{2}{*}{ Congestão } & Controle & -1 & -1 & -1 & -2 & -2 & -2 & -2 & -1 & -2 & -2 & \multirow{2}{*}{0,130} \\
\hline & Experimento & -2 & -2 & -2 & -2 & -2 & -2 & -2 & -2 & -2 & -1 & \\
\hline \multirow[t]{2}{*}{ Edema } & Controle & -2 & -2 & -2 & -2 & -1 & -3 & -3 & -1 & -1 & -1 & \multirow{2}{*}{0,427} \\
\hline & Experimento & -2 & -2 & -2 & -2 & -2 & -2 & -2 & -2 & -2 & -1 & \\
\hline \multirow[t]{2}{*}{ Polimorfonucleares } & Controle & -2 & -2 & -1 & -2 & -1 & -1 & -3 & -2 & -2 & -3 & \multirow{2}{*}{1} \\
\hline & Experimento & -3 & -3 & -1 & -2 & -3 & -3 & -2 & -1 & -2 & -1 & \\
\hline \multirow[t]{2}{*}{ Mononucleares } & Controle & 0 & 0 & 1 & 0 & 0 & 0 & 1 & 0 & 0 & 0 & \multirow{2}{*}{$0,023^{*}$} \\
\hline & Experimento & 1 & 1 & 1 & 1 & 1 & 1 & 1 & 0 & 0 & 1 & \\
\hline \multirow{2}{*}{$\begin{array}{l}\text { Prolif. } \\
\text { fibroblástica }\end{array}$} & Controle & 0 & 0 & 0 & 0 & 0 & 0 & 0 & 0 & 0 & 0 & \multirow{2}{*}{$0,059^{*}$} \\
\hline & Experimento & 0 & 0 & 1 & 0 & 0 & 0 & 1 & 1 & 1 & 1 & \\
\hline \multirow[t]{2}{*}{ Angiogênese } & Controle & 0 & 0 & 0 & 0 & 0 & 0 & 0 & 0 & 0 & 0 & \multirow{2}{*}{$0,059^{*}$} \\
\hline & Experimento & 0 & 0 & 1 & 0 & 0 & 0 & 1 & 1 & 1 & 1 & \\
\hline \multirow[t]{2}{*}{ Colagenização } & Controle & 0 & 0 & 0 & 0 & 0 & 0 & 0 & 0 & 0 & 0 & \multirow{2}{*}{1} \\
\hline & Experimento & 0 & 0 & 0 & 0 & 0 & 0 & 0 & 0 & 0 & 0 & \\
\hline \multirow[t]{2}{*}{ Somatório } & Controle & -5 & -5 & -4 & -5 & -4 & -4 & -7 & -4 & -5 & -6 & \multirow{2}{*}{--} \\
\hline & Experimento & -6 & -6 & -4 & -5 & -6 & -6 & -3 & -3 & -4 & 0 & \\
\hline \multirow[t]{2}{*}{ Inflamação } & Controle & $\mathrm{A}$ & A & A & A & $\mathrm{A}$ & A & A & A & A & A & \multirow{2}{*}{0,705} \\
\hline & Experimento & $\mathrm{A}$ & $\mathrm{A}$ & A & $\mathrm{A}$ & $\mathrm{A}$ & A & $\mathrm{A}$ & $\mathrm{A}$ & $\mathrm{A}$ & B & \\
\hline
\end{tabular}

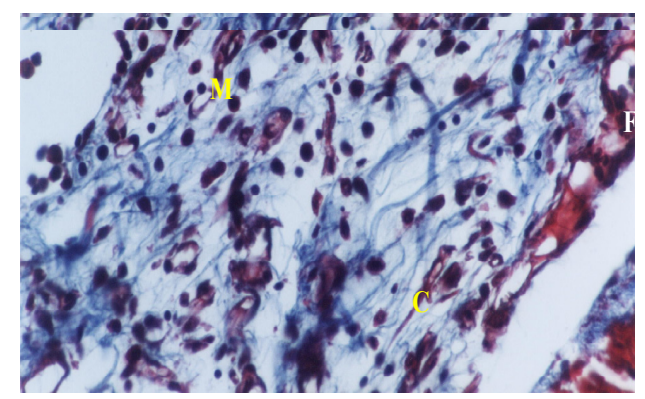

FIGURA 5 - Fotomicrografia de cólon do grupo controle no $7^{\circ}$ dia (Tricômio de Masson, 400x.). Nota: Observa-se inflamação crônica com mononucleares (M), fibroblastos (F) e colágeno $(\mathrm{C})$.

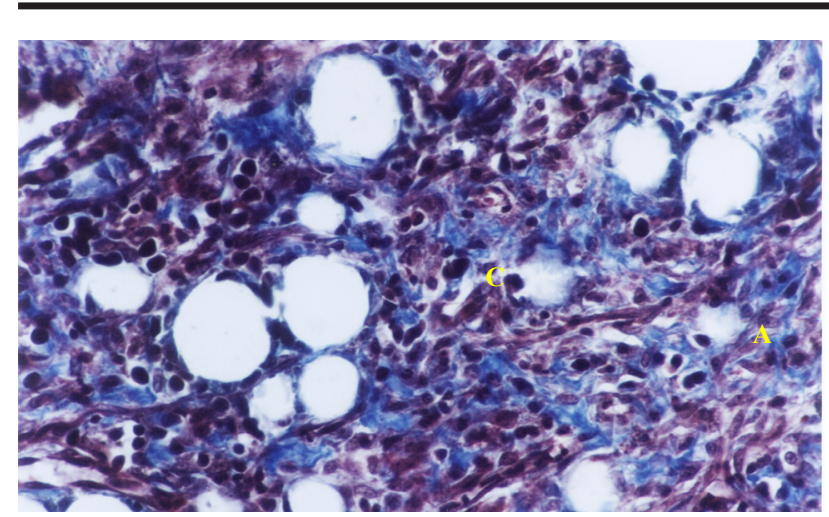

FIGURA 6 - Fotomicrografia de cólon do grupo experimento no $7^{\circ}$ dia (Tricômio de Masson, 400x.). Nota: Observa-se inflamação crônica com angiogênese (A) e colágeno (C).
TABELA 5 - Variáveis da análise histológica do $7^{\circ}$ dia

\begin{tabular}{|c|c|c|c|c|c|c|c|c|c|c|c|c|}
\hline $\begin{array}{l}\text { Variável } \\
\text { histológica }\end{array}$ & Grupo & R1 & R2 & R3 & R4 & R5 & R6 & R7 & R8 & R9 & R10 & $\mathrm{P}$ \\
\hline \multirow[t]{2}{*}{ Congestão } & Controle & -1 & -1 & -1 & -1 & -1 & -1 & -1 & 0 & -1 & 1 & \multirow{2}{*}{$0,002 *$} \\
\hline & Experimento & 0 & 0 & 0 & -1 & 0 & 0 & 0 & 0 & 0 & 0 & \\
\hline \multirow[t]{2}{*}{ Edema } & Controle & -2 & -2 & -2 & -2 & -2 & -1 & -1 & -1 & -2 & -1 & \multirow{2}{*}{$0,002 *$} \\
\hline & Experimento & 0 & 0 & 0 & -1 & 0 & -1 & -1 & -1 & -2 & -1 & \\
\hline \multirow[t]{2}{*}{ Polimorfonucleares } & Controle & -2 & -2 & -2 & -2 & -2 & -2 & -1 & -1 & -2 & -1 & \multirow{2}{*}{$0,008^{*}$} \\
\hline & Experimento & -1 & -1 & -1 & -1 & -1 & -1 & -1 & -1 & -1 & -1 & \\
\hline \multirow[t]{2}{*}{ Mononucleares } & Controle & 0 & 0 & 0 & 1 & 1 & 0 & 2 & 3 & 1 & 1 & \multirow{2}{*}{$0,001 *$} \\
\hline & Experimento & 2 & 2 & 3 & 3 & 2 & 3 & 3 & 3 & 3 & 3 & \\
\hline \multirow{2}{*}{$\begin{array}{l}\text { Prolif. } \\
\text { fibroblástica }\end{array}$} & Controle & 0 & 0 & 0 & 1 & 0 & 1 & 2 & 3 & 0 & 1 & \multirow{2}{*}{$0,008^{*}$} \\
\hline & Experimento & 2 & 1 & 2 & 2 & 2 & 2 & 2 & 2 & 3 & 3 & \\
\hline \multirow[t]{2}{*}{ Angiogênese } & Controle & 0 & 0 & 0 & 1 & 1 & 0 & 0 & 2 & 0 & 0 & \multirow{2}{*}{$0,017^{*}$} \\
\hline & Experimento & 0 & 1 & 2 & 1 & 1 & 1 & 2 & 2 & 2 & 1 & \\
\hline \multirow[t]{2}{*}{ Colagenização } & Controle & 0 & 0 & 0 & 0 & 0 & 0 & 0 & 2 & 0 & 0 & \multirow{2}{*}{$0,007^{*}$} \\
\hline & Experimento & 1 & 0 & 1 & 1 & 1 & 1 & 1 & 1 & 1 & 1 & \\
\hline \multirow[t]{2}{*}{ Somatório } & Controle & -5 & -5 & -5 & -2 & -3 & -3 & 1 & 8 & -4 & -1 & \\
\hline & Experimento & 4 & 3 & 7 & 4 & 5 & 5 & 6 & 6 & 7 & 7 & \\
\hline \multirow[t]{2}{*}{ Inflamação } & Controle & A & A & A & B & A & A & $\mathrm{C}$ & $\mathrm{C}$ & A & B & \multirow{2}{*}{$0,002^{*}$} \\
\hline & Experimento & $\mathrm{C}$ & $\mathrm{C}$ & $\mathrm{C}$ & $\mathrm{C}$ & $\mathrm{C}$ & $\mathrm{C}$ & $\mathrm{C}$ & $\mathrm{C}$ & $\mathrm{C}$ & $\mathrm{C}$ & \\
\hline
\end{tabular}
controle e experiment

\section{Discussão}

As anastomoses término-terminais foram realizadas com a colocação de seis pontos separados, plano único e inversão da mucosa por ser prática e segura e exibir menor índice de estenose, resultando em luz mais ampla e menos danos das bordas teciduais. O mesmo foi considerado por Thornton ${ }^{11}$, estudando cicatrização no trato gastrointestinal com relação à técnica cirúrgica. Este trabalho ratifica os mesmos achados em outros estudos por não ter apresentado estenose, deiscência ou fístula.

Escolheu-se como períodos para a eutanásia dos animais o $3^{\circ}$ e $7^{\circ}$ dias de pós-operatório devido a que no $3^{\circ}$ permite-se o estudo da cicatrização na sua fase inicial ou inflamatória aguda, em que a taxa global de lise do colágeno é maior do que a síntese, em decorrência da ação da colagenase e no $7^{\circ}$ dia porque a presença do colágeno (que caracteriza a fase crônica da cicatrização) começa a ser notada com mais intensidade (Corsi et al. ${ }^{2}$ ).

Todos os 40 animais evoluíram bem em relação ao procedimento cirúrgico revelado pela manutenção do peso, da atividade motora e disposição para alimentação. As complicações apresentadas foram infecção discreta do sítio cirúrgico, deiscência cutânea superficial e presença de aderências frouxas entre as alças intestinais e a parede abdominal; essas últimas com significância estatística nos animais do grupo do $3^{\circ}$ dia.

Tabushi $^{12}$, realizando experimentalmente cecorrafia em plano único com polipropileno e poliglecaprone em $25 \mathrm{em}$ ratos, confirma também a presença de aderências frouxas entre a parede abdominal, omento e alças intestinais. Nomura ${ }^{13}$ em estudo experimental comparativo de cicatrização de suturas em ceco de ratos, constatou presença de aderências intra-abdominais pós-operatórias. Este fato demonstra mais uma vez que as aderências estão mais ligadas com a atividade reacional dos tecidos e a ação do fator de crescimento transformante beta (TGF) das plaquetas e liberado na fase inflamatória aguda, do que propriamente com o tipo de fio utilizado. Ressalte-se que este fator, além da indução 
da angiogênese e proliferação fibroblástica, produz maior formação de aderências no peritôneo.

Os métodos quantitativos para estudo experimental das anastomoses cirúrgicas incluem análise mecânica e bioquímica. Os parâmetros mecânicos mais usados são: força de ruptura (breaking strength) e pressão de explosão (bursting pressure). A força de ruptura é medida de resistência da parede intestinal contra a força aplicada na direção longitudinal da anastomose. Vários trabalhos têm apresentado dados contraditórios Weiber et al. apud Mânsson et al. ${ }^{14}$ afirmam ser a força de ruptura constante a partir do $4^{\circ}$ dia de anastomose colônica, enquanto outros autores (Hendriks) ${ }^{15}$ relatam que durante os três ou quatro primeiros dias permanecem baixas.

Dentre os fatores responsáveis pela alterações de dados na avaliação da força de ruptura, citam-se técnica cirúrgica imperfeita (distância entre as bordas da ferida e a colocação dos pontos cirúrgicos) e a pressão intra-luminar exercida somente no sentido longitudinal, sendo inócua no circular.

A pressão de explosão é definida como aquela que determina a ruptura ou explosão da anastomose. Ballantine ${ }^{15}$, Brasken $^{16}$, Hendriks ${ }^{14}$, Ikeuchi et al., ${ }^{15}$, consideram que ela aumenta progressivamente após a formação da anastomose colônica e é útil para mensurar mudanças precoces durante a primeira semana após a operação, diferentemente da força de ruptura que não apresenta mudanças nos primeiros três dias após cicatrização da anastomose.

Os índices de pressão de insuflação de ar atmosférico nos animais do grupo do $3^{\circ}$ dia (controle e experimento) apresentaram índices baixos em relação aos animais do grupo do $7^{\circ}$ dia (controle e experimento). Estes resultados confirmam a tendência apresentada na literatura, repetindo o comportamento padrão conhecido de baixa resistência à insuflação de ar no período de maior colagenólise, isto é, $3^{\circ}$ dia de pós-operatório.

Contudo, apesar de que os índices de pressão de insuflação de ar atmosférico fossem mais elevados nos animais do grupo do $7^{\circ}$ dia quando comparados com os do $3^{\circ}$ dia, a análise estatística não apresentou diferença significante.

Conquanto não tenham ocorrido neste estudo complicações relacionadas à deiscência, ou fístula anastomótica, é importante a observação de que é neste período de três dias que ocorrem vazamentos na linha anastomótica. Ele é decorrente de intensa atividade colagenolítica, sendo os granulócitos as células responsáveis por grau significativo deste fenômeno.

Hendriks ${ }^{14}$, Nomura ${ }^{19}$, Mansson et al. ${ }^{18}$, estudando cicatrização de anastomoses colônicas, utilizando como parâmetro mecânico teste de avaliação tensiométrica, encontraram comportamento similar aos apresentados neste trabalho.

Neste estudo, estabeleceu-se a classificação da cicatrização em aguda, sub-aguda e crônica, baseada na análise do índice histológico obtido, em que a fase aguda é caracterizada pela presença de congestão, edema e polimorfonucleares; a fase sub-aguda à presença de mononucleares, proliferação fibroblástica e angiogênese; e a colagenização definindo a fase crônica. Também foram estabelecidos índices numéricos negativos para a $1^{\mathrm{a}}$ fase de inflamação ou inflamação aguda e índices numéricos positivos para a $3^{\mathrm{a}}$ fase ou fase crônica da cicatrização. A importância deste modelo de classificação é que, além de colocar em evidência os eventos bioquímicos-celulares de cada fase da cicatrização, ao estabelecerem análise numerizada, possibilitam também estudo estatístico mais acurado.

A análise microscópica do $3^{\circ}$ dia mostrou que todos os animais do grupo controle e experimento apresentaram congestão, edema e polimorfonucleares, elementos que caracterizam a inflamação aguda. Observou-se, também, nesta fase nos animais do grupo experimento a presença de mononucleares, proliferação fibroblástica e angiogênese, sendo que os mononucleares mostraram significância estatística $(\mathrm{p}=0,023)$, já denotando possível ação do extrato aquoso do Babaçu.

É importante assinalar a ação dos mononucleares, células que produzem e liberam monocinas, controlam a formação do tecido de granulação, influenciam a angiogênese, que é sempre ativada pela hipóxia tecidual e diminuição do $\mathrm{pH}$ da ferida, além de promoverem quimiotaxia induzindo a migração e proliferação fibroblástica.

A presença de angiogênese e fibroplasia, nesta fase inicial da cicatrização, ou fase inflamatória aguda evidenciada neste estudo, é decorrente, além da ação dos mononucleares, da ativação de macrófagos e plaquetas que liberam citocinas e fatores de crescimento. Brasken ${ }^{18,19,20}$, constataram em seus experimentos, a presença de angiogênese e proliferação fibroblástica na fase inflamatória aguda.

A análise microscópica do $7^{\circ}$ dia evidencia a presença de inflamação crônica em todos os animais do grupo experimento, enquanto que no grupo controle somente um animal a apresentou. A inflamação crônica constatada neste estudo corresponde à $3^{\mathrm{a}}$ fase da cicatrização, também denominada fase de maturação ou remodelagem, em que a presença do colágeno é evento importante. No trato gastrointestinal, o colágeno é sintetizado por fibroblastos e células musculares lisas, predominando na cicatriz o colágeno tipo I (68\%) que desempenha importante papel na força tênsil da cicatriz.

Ainda no grupo experimento, observou-se que todos os parâmetros que caracterizam a fase aguda (congestão, edema e polimorfonucleares) e sub-aguda (mononucleares, proliferação fibroblástica e angiogênese) apresentaram significância estatística.

\section{Conclusão}

1) Não houve diferença significante na avaliação tensiométrica entre os grupos controle e experimento.

2) Todos os parâmetros histológicos estudados mostraram-se positivos na cicatrização com o grupo experimento no $7^{\circ}$ dia, evidenciando efeito favorável do extrato aquoso do mesocarpo do Babaçu em nível microscópico do processo de cicatrização de anastomoses colônicas.

\section{Referências}

1. Amorim, J. de A. Fitoterapia Popular e Saúde da Comunidade. [Tese- Doutorado]. São Paulo: Faculdade 
de Saúde Pública, Universidade de São Paulo,1999.

2. Ballantine GH. The experimental basic of intestinal suturing: effect of surgical technique, inflammation, and infection on enteric wound healing. Dis Colon Rectum. 1984; 27:61-71.

3. Bevilaqua RG; Chapchap P, Almeida CG. de. Cicatrização. In: Allgower M, Bevilaqua RG, editores. Manual de Cirurgia. São Paulo: Pedagógica Universitária, 1981.

4. Brasken P, Renval S. Local energy metabolism in healing colon anastomosis. Acta Chir Scand 1990;156: 56570.

5. Brito MVH, Brito NMB, Almeida AJB, Santos MRLC. Vaporizador artesanal de éter para cirurgia experimental em pequenos roedores. Acta Cir Bras. 1998;13:3-7.

6. Brito RM F. Avaliação de auto-reatividade induzida pelo tratamento com o Babaçu. [Monografia- Especialização]. São Luis: Setor de Ciências Biológicas, Universidade Federal do Maranhão; 2001.

7. Corsi RCC, Corsi PR, Pirana S, Muraco FAE, Jorge D. Wound healing: a Review. Rev Col Bras Cir. 1994;84:1724.

8. Coskun I, Irfanoglu ME, Hatipoglu AR. The preventive of carboxymetil cellulose (CCM) on the intraperitoneal adhesion in rats. Turk J Surg. 1992; 8:93-6.

9. Chagas AP. Atividade do mesocarpo do Babaçu (Orbignya phalerata) sobre o desenvolvimento tumoral e a população de linfócitos B. [Monografia]. São Luis: Departamento de Biologia, Centro de Ciências Biológicas e de Saúde, Universidade Federal do Maranhão; 2001.

10. Clark RAF, Henson PM. The molecular and cellular biology of wound repair. New York: Plenum Press, 1988.

11. Fatouros MS, Vekinis G, Bourantas KL, Mylonakis EP, Scopelitou AS, Malamou-Mitsis VD, Kappas AM. Influence of growt factors eritropoethin and granulocite macrophage colony stimulating factor on mechanical streng and healing of colon anastomoses in rats. Eur J Surg. 65: 986-92, 1999.
12. Felix VN, Yogi I, Camerini ESN. O organismo, a ferida e o processo de cicatrização. Rev Bras Med. 1990; 47:32530.

13. Greca FH, Biondo-Simões MLP, Collaço LM, Martins VDM, Tolazzi, ARD, Gasparetto EL, Santos EAA. A ação dos ácidos graxos de cadeia curta na cicatrização de anastomoses colônicas: estudo experimental em ratos. Acta Cir Bras. 2000;15:1-9.

14. Hendriks T, Mastboom WJB. Healing of experimental intestinal anastomoses: parameters for repair. Dis Colon Rectum 1990;3:891-901.

15. Ikeuchi, D. Correlation of tensile strenght with bursting pressure in the evaluation of intestinal anastomosis. Dig Surg. 1999; 16:.478- 86.

16. Landmann J. As medicinas alternativas: mito, embuste ou ciência. Rio de Janeiro: Guanabara Koogan, 1989.

17. Maia MBS. Estudo da atividade anti-inflamatória e outros efeitos farmacológicos relacionadas à de Orbignya phalerata mart. [Dissertação- Mestrado]. Fortaleza: Departamento de Fisiologia e Farmacologia . Universidade Federal do Ceará; 1987.

18. Mansson P, Zhang Xiao Wei, Jppsson, B, Thorlacius $\mathrm{H}$. Anastomotic healing in the rat: comparison between a radiological method, breaking strength and bursting pressure. Int J Colorectal Dis. 2002;17:420-5.

19. Nomura LM. Estudo experimental comparativo da cicatrização de sutura em ceco de ratos, utilizando os fios de polipropilene, poliglecaprone 25 e glicomer 60. [Dissertação -Mestrado]. Curitiba: Departamento de Cirurgia do Hospital Universitário. Evangélico de Curitiba, Faculdade Evangélica de Medicina do Paraná;1999.

20. Rosental FRT, Espíndola AMC. O amido do coco de Babaçu, algumas propriedades das grânulas e das pastas. Rev Bras Tecnol. 1975: 6-29, 1975.

\section{Correspondência}

Raimundo Nonato Baldez

Hospital Universitário Presidente Dutra

Rua Barão de Itapary, 227 - Centro - São Luis-MA

CEP: $65020-070$

Tel: (98) 2109-1000
Conflito de interesse: nenhum

Fonte de financiamento: Capes

Recebimento: 08/01/2005

Revisão: 23/06/2005

Aprovação: 11/06/2006

\section{Como citar este artigo}

Baldez RN, Malafaia O, Czeczko NG, Martins NLP, Ferreira LM, Ribas CAPM, Salles Jr G, Del-Claro RP, Santos LOM, Graça-Neto L, Araújo LRR. Análise da cicatrização do cólon com uso do extrato aquoso da Orbignya phalerata (Babaçu) em ratos. Acta Cir Bras. [periódico na Internet] 2006; Suppl 2:31-38. Disponível em URL: http://www.scielo.br/acb

Figuras coloridas disponíveis em http://www.scielo.br/acb 